\title{
Senecio farinifer y S. madariagae (Asteraceae, Senecioneae), NUEVOS REGISTROS PARA LA FLORA ARGENTINA
}

\author{
MARCELO P. HERNÁNDEZ $Z^{1,2^{*}}$, SUSANA E. FREIRE ${ }^{3}$ y DANIEL A. GIULIANO ${ }^{1}$
}

\begin{abstract}
Resumen: En el presente trabajo se registra por primera vez para la flora argentina la presencia de Senecio farinifer Hook. \& Arn. y S. madariagae Phil. (Asteraceae-Senecioneae). Se presenta una descripción de cada especie incluyendo microcaracteres vegetativos y reproductivos.
\end{abstract}

Palabras clave: Senecio farinifer, S. madariagae, Asteraceae, Salta, San Juan.

\begin{abstract}
Summary: Senecio farinifer and Senecio madariagae (Asteraceae-Senecioneae), new records for Argentine flora. This paper records for the first time the presence in the Argentine flora of Senecio farinifer Hook. \& Arn. and S. madariagae Phil. (Asteraceae-Senecioneae). A description, including vegetative and reproductive microcharacters, is provided.
\end{abstract}

Key words: Senecio farinifer, S. madariagae, Asteraceae, Salta, San Juan.

\section{INTRODUCCIÓN}

El género Senecio L. s.str. (Asteraceae), con aproximadamente 1000 especies prácticamente cosmopolitas (Pelser et al., 2007), constituye una de las unidades sistemáticas más grandes dentro de las Angiospermas en general y de las Asteráceas en particular. La mayor concentración de especies del género se halla en las regiones montañosas de América, África y Asia, estando pobremente representado en las selvas ecuatoriales.

La subserie Polyphylli Giuliano, M. P. Hernández \& S. E. Freire (Giuliano et al., 2017) comprende 23 especies que crecen principalmente en la región andina de Argentina y Chile (Cabrera, 1949, 1985;

\footnotetext{
1 Área de Botánica, Departamento de Ciencias Biológicas, Facultad de Ciencias Agrarias y Forestales, Universidad Nacional de La Plata, Avda. 60 entre 116 y 118, 1900 La Plata, Argentina.

2 LAMCE (Laboratorio de Morfología Comparada de Espermatófitas), Área de Botánica, Departamento de Ciencias Biológicas, Facultad de Ciencias Agrarias y Forestales, Universidad Nacional de La Plata, Avda. 60 entre 116 y 118, 1900 La Plata, Argentina.

${ }^{3}$ Instituto de Botánica Darwinion, Casilla de Correo 22, Labardén 200, San Isidro (B1642HYD), Buenos Aires, Argentina.

*E-Mail: mphciencia@yahoo.com
}

Cabrera et al., 1999; Freire et al., 2014). Dentro de esta subserie, Senecio farinifer Hook \& Arn. y S. madariagae Phil. se distribuyen en el centro y norte de Chile respectivamente.

Recientes viajes del primer autor (M.P.H.) a la provincia de San Juan en febrero de 2016, como parte de su trabajo de tesis doctoral (Hernández, 2016), han permitido hallar ejemplares que se corresponden con la descripción de Senecio farinifer. Asimismo, la revisión del material de herbario facilitó la identificación de un ejemplar de $S$. madariagae procedente de la provincia de Salta.

El objetivo del presente trabajo es dar a conocer el primer registro de Senecio farinifer y $S$. madariagae para la Argentina. Se describen ambas especies incluyendo sinónimos, citas de iconografías y datos acerca de la distribución, hábitat y fenología; asimismo, se las diferencia de las restantes especies de la subserie que crecen en las respectivas provincias.

\section{Resultados}

Senecio farinifer Hook. \& Arn., J. Bot. [Hooker] 3: 331. 1841. TIPO. Chile. Near Viña de la Mar, Bridges 223 (K000527689!); Valparaíso, H. Cuming 583 (E00251538!). 
Senecio belophyllus J. Rémy, in Gay, Fl. Chil. 4: 164. 1849. TIPO: Chile. Sin localidad consignada, 1839, C. Gay 120 (BR0000005528097!, P02296705!, P02296706!, P02296707!).

Senecio linariifolius Poepp. ex DC. var. longifolia Phil., Anales Univ. Chile 88: 245. 1894. TIPO: Chile. Quillota, Cerro de la Campanita, XII-1883, F. Philippi s.n. (SGO, SI043417!).

Senecio apricus Phil., Anales Univ. Chile 88: 261. 1894. TIPO: Chile. Estancia Mercedes, XI1888, R. Philippi s.n. (SGO060635!).

Sufrútices lanuginosos, densamente ramosos desde la base, de 20-40 $\mathrm{cm}$ de altura. Tallos delgados, laxamente hojosos, muy divididos, con pelos glandulosos blancos. Hojas alternas, glabras, glabrescentes a pubescentes (levemente lanuginosas) y glandulosas en ambas caras, lineares, de 15-55 × 1-3 mm, levemente atenuadas en la base, agudas en el ápice, las superiores muy cortamente sagitadas, enteras, más o menos revolutas en el margen (muy rara vez con algún diente). Capítulos radiados, solitarios en el ápice de las ramas escapiformes, casi desnudas en su parte superior. Involucro anchamente acampanado, de 5,5-9 × 7-10 mm, caliculado, bractéolas del calículo 3-5 (excepcionalmente 0-1), lineares, alcanzando menos de la mitad del largo del involucro. Filarios 16-20, linear-lanceolados, atenuados y penicilados en el ápice, con pelos glandulosos blancos y crespos en el dorso. Flores dimorfas; las marginales 12-20, pistiladas, con corolas amarillas liguladas, lígula oblonga, 2-3-dentada, de 7-9 × 2-3 mm, tubo ca. 3,5 mm de largo; las del centro numerosas, perfectas, con corolas amarillas tubulosas, de 5-7 mm de largo, 5-dentadas en el ápice, dientes triangulares de 0,5 $\mathrm{mm}$ de largo, con vena media o sin ésta; anteras con collares balusteriformes de menos de $1 \mathrm{~mm}$ de largo, apéndices conectivales ovados; ramas del estilo truncadas en el ápice donde llevan una corona de pelos colectores. Aquenios cilindroides, de 1-2 mm de largo, 8-10-costados, densa y cortamente papiloso-pubescentes. Papus blanco, de 6-9 mm de largo. (Figs. 1, 2).

Iconografía. Cabrera (1949, p. 185, fig. 59).

Distribución y hábitat. Especie hasta ahora mencionada para el centro de Chile, extendiéndose en las montañas bajas, en las Regiones de Coquimbo, Valparaíso, Metropolitana de Santiago y Maule (Cabrera, 1949; Freire, 1999). La revisión del material de herbario llevada a cabo durante el desarrollo de la tesis doctoral ha permitido hallar ejemplares procedentes de las Regiones Libertador Bernardo O'Higgins y Araucanía, ampliando de esta manera su distribución en Chile. Asimismo, recientes colectas han permitido hallar ejemplares de esta especie en la provincia de San Juan, lo que constituye la primera cita de esta especie para la Flora Argentina. Vegeta en las provincias biogeográficas Altoandina (entre los 900-2200 m s.m.), Prepuneña (entre 1000 y 3400 m s.m.) y Chilena Central (Cabrera, 1976).

Fenología. Florece desde septiembre hasta marzo.

Observación. Hasta el momento, para la provincia de San Juan sólo se ha citado la presencia de una especie de Senecio perteneciente a la subserie Polyphylli, S. segethii Phil. (Tombesi \& Freire, 2013; Freire et al., 2014). Senecio farinifer se diferencia de $S$. segethii por su hábito sufruticoso de 20-40 cm de alto, hojas lineares y flores con corola ligulada amarilla (vs. hábito herbáceo de 7-10 cm de alto, hojas oblanceoladoespatuladas y flores con corola ligulada blanca).

Materiales adicionales examinados. ARGENTINA. Prov. San Juan: Dpto. Calingasta, Calingasta rumbo a Iglesia, a orillas de la ruta

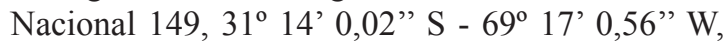
10-II-2016, Hernández 203 (LPAG). Dpto. Iglesia: Ruta Nacional 149, en la entrada a Iglesia, cerca del Santuario de Santa Gemma, a $50 \mathrm{~m}$ de la ruta, $30^{\circ} 37^{\prime} 57,49$ ' S - 69 5' 8,71' W, 11-II-2016, Hernández 204 (LPAG). Dpto. Jáchal: de Jáchal rumbo al Parque Nacional Talampaya, por Ruta Nacional 40, pasando el Río Jáchal, 29 50' 33, 57” S - 68 25' 57,22” W, 12-II-2016, Hernández 205 (LPAG); Huaco rumbo al Parque Nacional Ischigualasto, ruta 150 , sobre barranca del camino, $30^{\circ} 13$ ' 6,59" S - 68 31' 47" W, 13-II-2016, Hernández 206 (LPAG).

Senecio madariagae Phil., Anales Mus. Nac. Santiago de Chile Secc. 2, Bot. 8: 45. 1891. TIPO: Chile. Tarapacá, inter Amincha et 

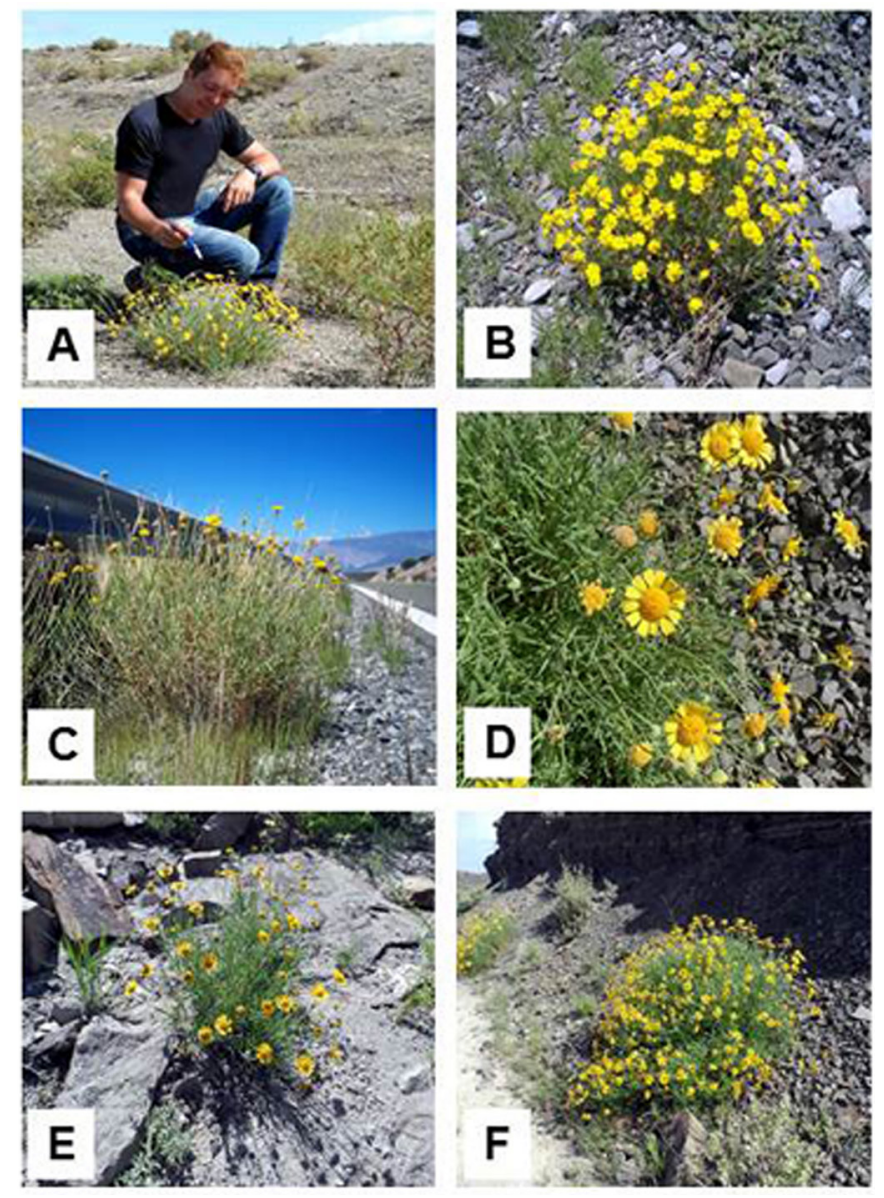

Fig. 1. Senecio farinifer. Individuos localizados en la provincia de San Juan: A: Dpto. Calingasta. B y C: Dpto. Iglesia. D, E y F: Dpto. Jáchal.

Paroma, 25-II-1885, F. Philippi s.n. (lectotipo, SGO44483!, designado por M. P. Hernández \& D. A. Giuliano, Bol. Soc. Argent. Bot. 49(1): 78. 2014; isolectotipos, B, foto Field Museum $\mathrm{n}^{\circ}$ 15639!, K000497901!, LP063405!, SGO60740!).

Arbustitos glabros o ligeramente lanosos en las partes jóvenes, densamente ramosos, de $30 \mathrm{~cm}$ de altura. Tallos algo ondulados, estriados, laxamente hojosos. Hojas alternas, sésiles, carnosas, las jóvenes lanuginosas, las adultas glabras a glabrescentes en la cara adaxial, glabras en la cara abaxial, oblongo-lineares, de 7-15 × 1-2 mm, ensanchadas y semiabrazadoras en la base, obtusas en el ápice, enteras o con 1-2 dientes a cada lado del margen, de 1-2 $\mathrm{mm}$ de largo. Capítulos radiados, solitarios o agrupados de a 2-3, en el ápice de ramas, pedunculados, pedúnculos bracteados, de 1,5-4,5 $\mathrm{cm}$ de largo. Involucro anchamente acampanado, ecaliculado, de $8-10 \times 12-14 \mathrm{~mm}$, de igual largo que las flores del centro. Filarios 12-14, lanceolados, atenuados en la base y penicilados en el ápice, glabros en el dorso, de 8-10 × 1-3 mm. Flores dimorfas; las marginales 8-10, pistiladas, de corolas amarillas liguladas, lígula oblanceolada, de $5 \times 2 \mathrm{~mm}$, 2-3-dentada en el ápice, dientes triangulares de 0,4-0,5 $\mathrm{mm}$ de largo, tubo de 5-5,5 $\mathrm{mm}$ de largo; flores del centro numerosas, perfectas, corolas amarillas tubulosas, de 7-8,5 mm de largo, 5-dentadas en el ápice, dientes triangulares gruesos de 0,5-0,7 mm de largo, con nervadura central gruesa; anteras con collar balusteriforme, de menos de $1 \mathrm{~mm}$ de largo, apéndice conectival 
Bol. Soc. Argent. Bot. 53 (2) 2018

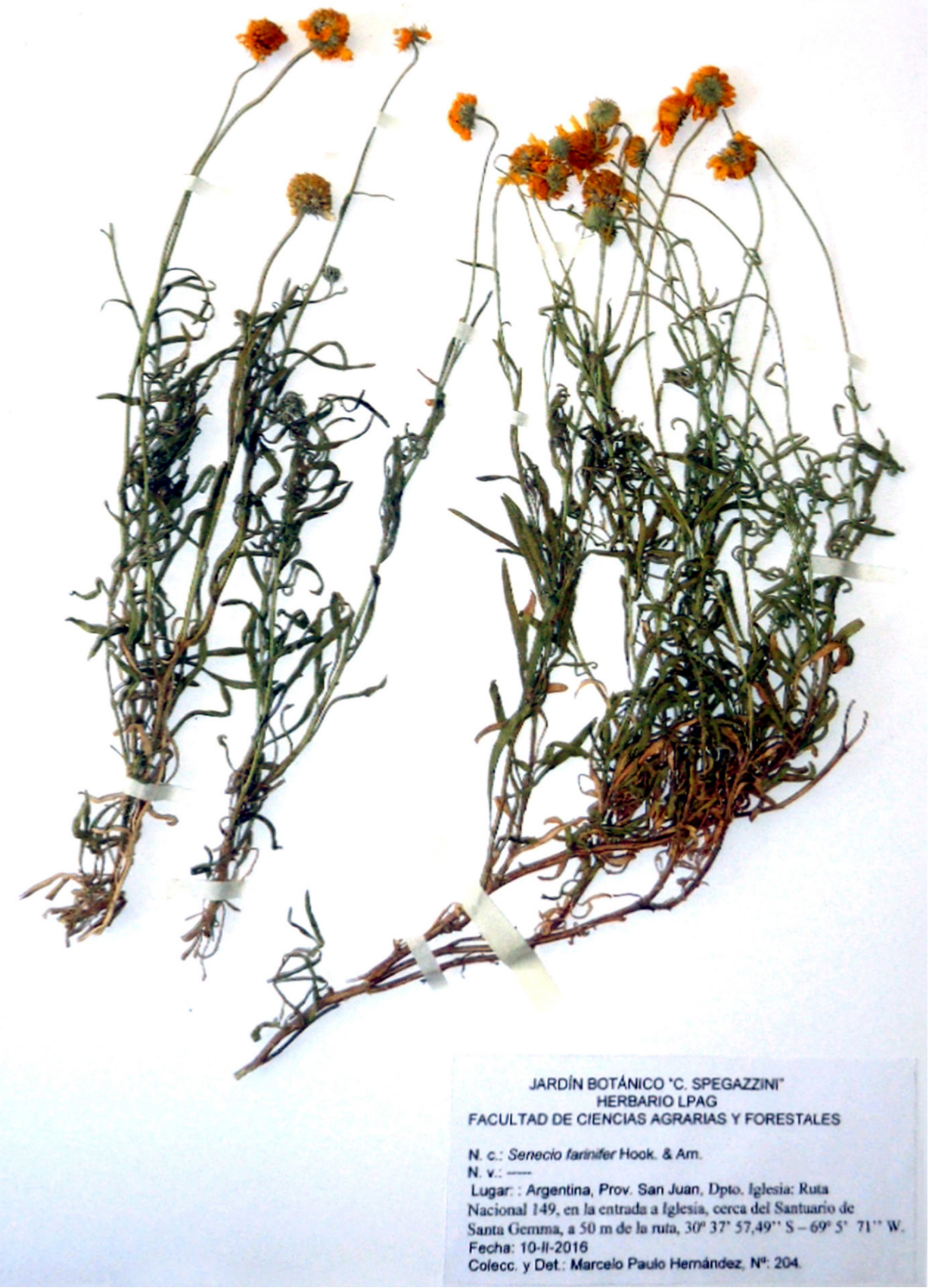

Fig. 2. Senecio farinifer. Ejemplar colectado en la provincia de San Juan (Hernández 204, LPAG).

ovado; ramas del estilo truncadas en el ápice donde llevan una corona de pelos colectores. Aquenios cilindroides, de 3-4 mm de largo, 8-10-costados, seríceo-pubescentes. Papus blanco, de 6-10 mm de largo. (Fig. 3).
Iconografia. Cabrera (1949, p. 187, Fig. 60).

Distribución y hábitat. Especie hasta ahora citada como endémica del norte de Chile (Cabrera, 1949; Freire, 1999). Sin embargo, la revisión del material de 


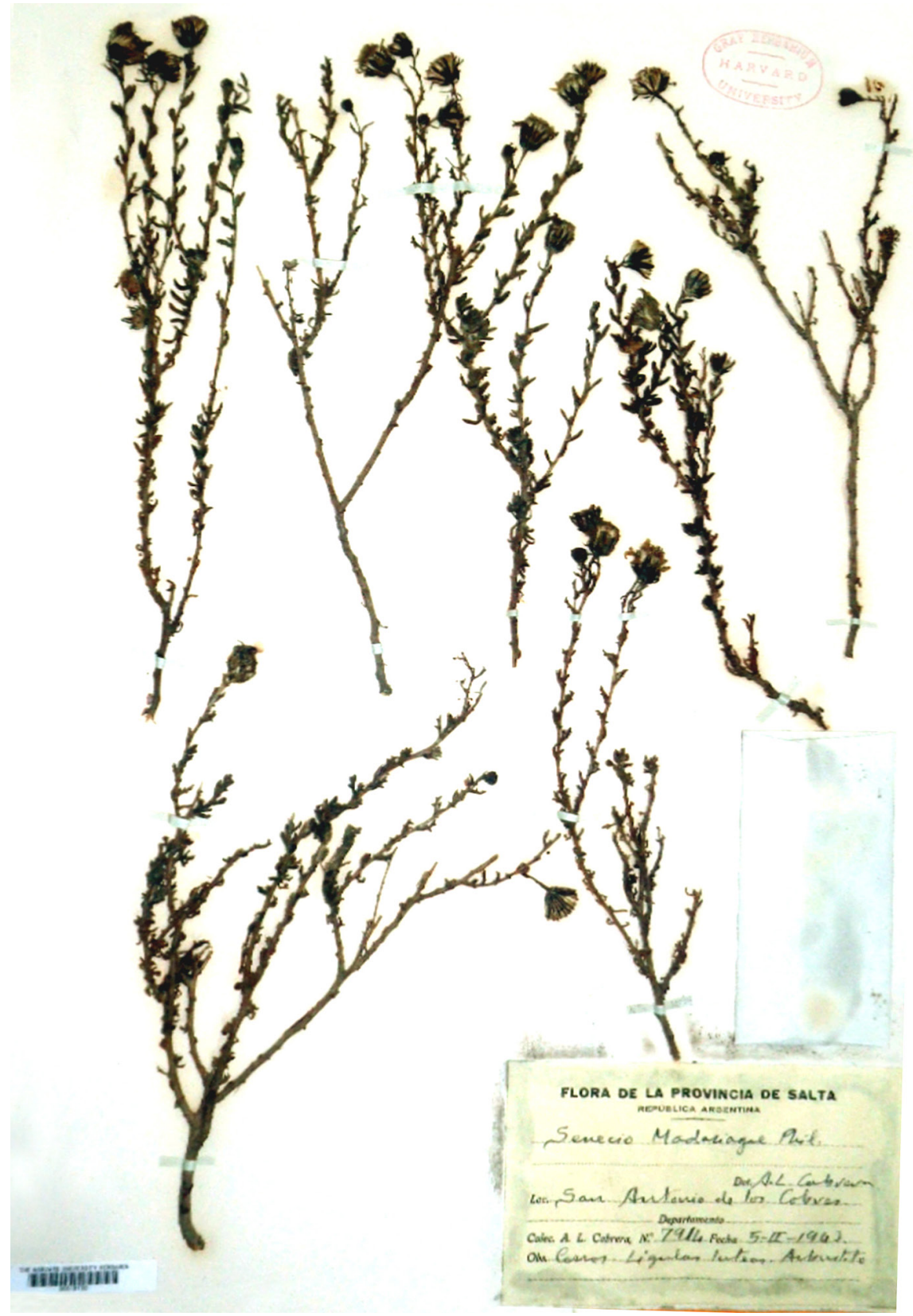

Fig. 3. Senecio madariagae. Ejemplar de herbario que constituye el nuevo registro de esta especie para la Flora Argentina, en la provincia de Salta (Cabrera 7914, GH).

herbario ha permitido hallar un ejemplar procedente de la provincia de Salta, el cual constituye la primera cita de esta especie para la Flora Argentina. Vegeta en la provincia biogeográfica Puneña entre 34003500 m s.m. (Cabrera, 1976).
Fenología. Ha sido colectada en flor desde enero hasta marzo, y en el mes de julio.

Observación. Hasta el momento, para la provincia de Salta sólo se ha citado la presencia de 
una especie de Senecio perteneciente a la subserie Polyphylli, S. punae Cabrera (Freire et al., 2014). Senecio madariagae se diferencia de $S$. punae por presentar hojas con margen entero o con 1-2 dientes a cada lado y capítulos ecaliculados (vs. hojas con margen con 2-3 lóbulos a cada lado y capítulos con numerosas brácteas del calículo).

Material adicional examinado. ARGENTINA. Prov. Salta: Depto. Los Andes, San Antonio de los Cobres, en los cerros, 05-II-1943, Cabrera 7914 (GH).

\section{Agradecimientos}

Al curador del herbario GH por el suministro del material correspondiente a Senecio madariagae y al Sr. Manuel Santos y la Sra. Nathalie Gelly, por su participación en el viaje de campaña a San Juan que nos permitió hallar los ejemplares de Senecio farinifer en esta provincia. Asimismo, a dos revisores anónimos por la lectura crítica del manuscrito.

\section{Biblografía}

CABRERA, A. L. 1949. El género Senecio en Chile. Lilloa 15: 27-501.

CABRERA A. L. 1976. Regiones fitogeográficas argentinas. En: Kugler W. F. (ed.). Enciclopedia Argentina de Agricultura y Jardinería. Tomo 2. 2 da. ed., ACME S. A. C. I. Buenos Aires. Argentina. Fascículo 1: 1-85.

CABRERA, A. L. 1985. El género Senecio (Compositae) en Bolivia. Darwiniana 26: 79-217.
CABRERA, A. L., S. E. FREIRE \& L. ARIZA ESPINAR. 1999. Género Senecio. En: Flora Fanerogámica Argentina Fascículo 62: 12-158. Proflora (Conicet).

FREIRE, S. E. 1999. Tribu Senecioneae. En: Zuloaga, F. O. \& O. Morrone (eds.), Catálogo de las Plantas Vasculares de la República Argentina II. Monog. Syst. Bot. Missouri Botanical Garden 74: 11541565.

FREIRE, S. E., L. ARIZA ESPINAR, L. SALOMÓN \& M. P. HERNÁNDEZ. 2014. Senecio. En: ZULOAGA, F. O., BELGRANO, M. J. \& ANTON, A. M. (eds.), Flora Argentina vol. 7(3): 27-220. Estudio Sigma S.R.L., Buenos. Aires, Argentina.

GIULIANO, D. A., M. P. HERNÁNDEZ \& S. E. FREIRE. 2017. Senecio subser. Polyphylli, a new name for Senecio subsect. Radiati (Asteraceae, Senecioneae) from Southern South America. Novon, 25: 43 .

HERNÁNDEZ, M. P. 2016. Revisión taxonómica y análisis cladístico de la subserie Radiati (Cabrera) Cabrera \& S.E. Freire del género Senecio (Asteraceae). Tesis Doctoral publicada on-line en SEDICI (Universidad Nacional de La Plata). 237 pp.

PELSER, P. B., B. NORDENSTAM, J. W. KADEREIT \& L. E. WATSON. 2007. An ITS phylogeny of tribe Senecioneae (Asteraceae) and a new delimitation of Senecio L. Taxon 56: 1077-1104.

TOMBESI, T. S. \& S. E. FREIRE. 2013. Senecio. En: KIESLING, R (dir.), Flora de San Juan, vol. 3-b: 115-158. Zeta Editores, Mendoza, Argentina.

Recibido el 15 de diciembre de 2017, aceptado el 30 de mayo de 2018. Editor: Massimiliano Dematteis. 